\title{
Comparison of neutron/gamma separation qualities of various organic scintillation materials
}

\author{
Zdeněk Matěj \\ Faculty of Informatics \\ Masaryk University \\ Brno, Czech Republic \\ Michal Koštál \\ Research Center Řež Ltd \\ Husinec- ̌̌ež, Czech Republic
}

\author{
Václav Přenosil \\ Faculty of Informatics \\ Masaryk University \\ Brno, Czech Republic
}

\author{
Filip Mravec \\ $\mathrm{VF}$, a.s. \\ Černá Hora, Czech Republic \\ František Kučera \\ Central European Institute of \\ Technology \\ University of Technology \\ Brno, Czech Republic \\ Zdeněk Kopecký \\ VF, a.s. \\ Černá Hora, Czech Republic
}

\author{
Aleš Jančář \\ $\mathrm{VF}$, a.s. \\ Černá Hora, Czech Republic
}

František Cvachovec

Faculty of Informatics

Masaryk University

Brno, Czech Republic

\author{
Jiří Čulen \\ $\mathrm{VF}$, a.s. \\ Černá Hora, Czech Republic
}

\begin{abstract}
In this work we compare the pulse-shape discrimination (PSD) properties of EJ-299-33A, BC-501A, stilbene, p-terphenyl and Hidex Aqualight in neutron field generated by the LVR-15 reactor with silicon filter utilization. Pulses from the scintillators are processed by Neutron-Gamma spectrometer. This spectrometric system with fast digitizer card contains two analog-digital (A/D) converters with a resolution of 12 bits and sampling frequency $500 \mathrm{MHz}$. For photomultiplier (PMT) linearity improvement active divider has been used. Measured data from scintillators have been processed using the integration method and compared. Results are presented.
\end{abstract}

Keywords- organic scintillators, neutron, gamma, impulse separation, FPGA, FoM, LVR-15

\section{INTRODUCTION}

The scintillators that enable the separation of neutrongamma radiation are widely used for measurements of mixed radiation fields. Several competing types of PSD capable scintillators were compared (EJ-299-33A, BC-501A, stilbene, p-terphenyl and Hidex Aqualight).The reactor LVR-15 with well-known radiation field has been used for measurements in the energy range 1 to $10 \mathrm{MeV}[1,2]$. Measured data have been processed using digital Neutron-Gamma spectrometer which we developed. The digital spectrometric system minimizes the drawbacks of analog devices and provides substantial simplification for the operation and setting of the system. The spectrometer is connected with a computer via Ethernet.

\section{EXPERIMENTAL SETUP}

Laboratory with experimental reactor LVR-15 has been used for the measurement, see Fig. 1.

We utilized well defined moderate neutron beam from the horizontal channel HK-1, see Fig. 2. Reactor thermal power output was $10 \mathrm{MW}$. Neutron beam has been moderated via 1 $\mathrm{m}$ wide silicon single crystal which provides spectrum with characteristic energy peaks, see unfolded spectrum in Fig. 3 [4].

The experimental arrangement is shown in Fig. 2. Influence of thermal neutrons and gammas was reduced via filter composed of ${ }^{6} \mathrm{Li}, \mathrm{Cd}, \mathrm{Bi}$ and $\mathrm{Pb}$.

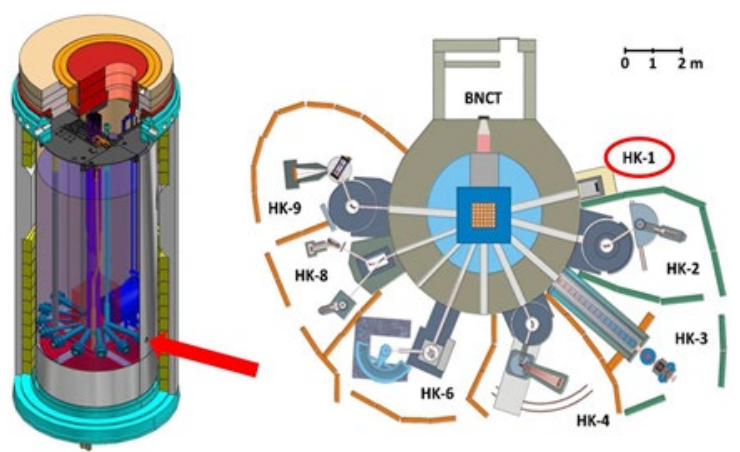

Fig. 1. - LVR-15 reactor (left) and its horizontal channels (right) [1]

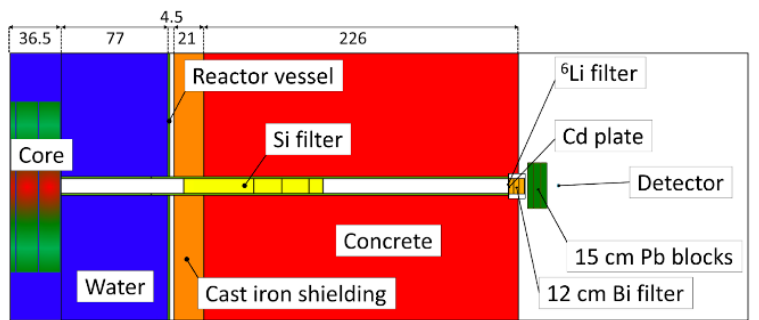

Fig. 2. The experimental arrangement in the reactor laboratory. The detectors were placed along the beam axis at $1.5 \mathrm{~m}$ distance from the silicon filter target [1].

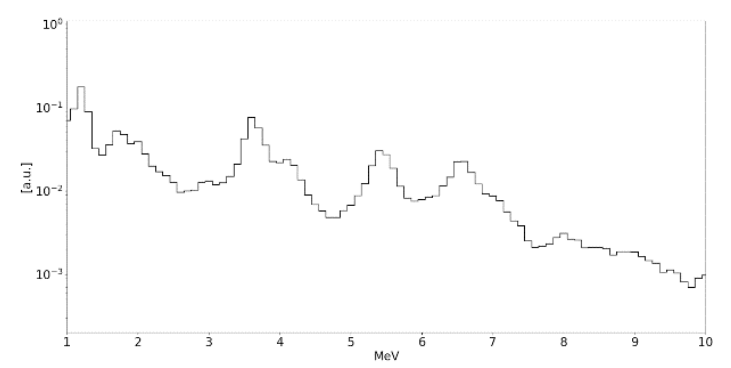

Fig. 3. Unfolded silicon moderated neutron spectrum measured with stilbene.

\section{RESULTS}

Integration method based on the pulse charge comparison was implemented in FPGA. Separation parameters for various 
scintillators calculated with this method are shown below in PSD matrices.

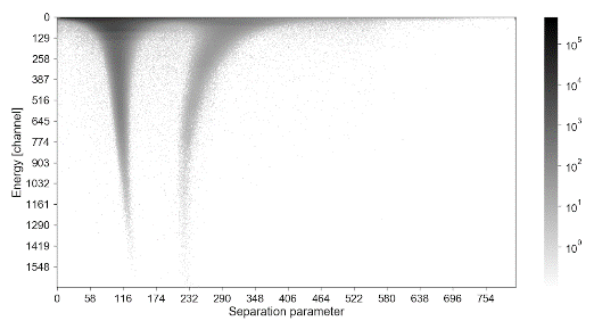

Fig. 4. Stilbene

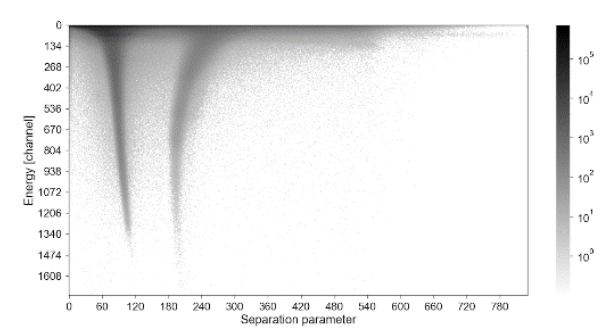

Fig. 5. BC-501A

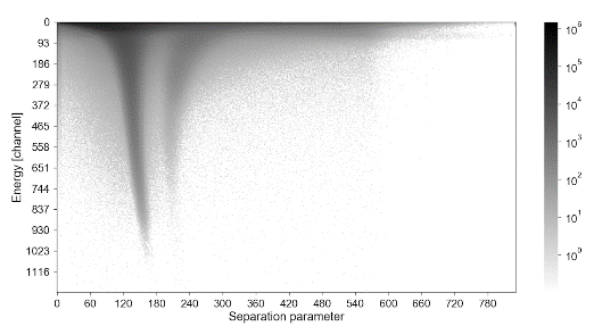

Fig. 6. EJ-299-33A

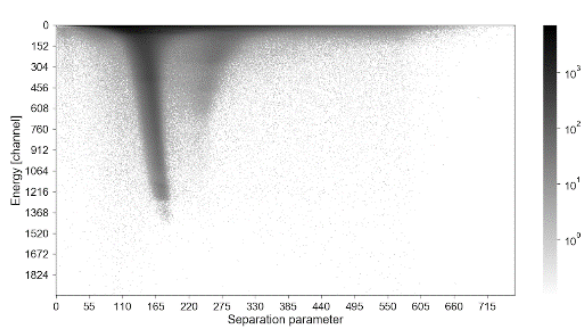

Fig. 7. P-terphenyl

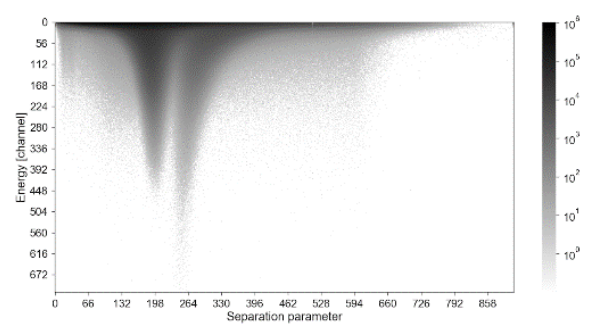

Fig. 8. Hidex Aqualight

Neutron-Gamma spectrometric system has been used for the measurements. The input analog signal from the detector was digitized by two fast $\mathrm{A} / \mathrm{D}$ converters working with a sampling frequency of $500 \mathrm{MHz}$. Digital signal processing is implemented in FPGA Xilinx Virtex-6.

\section{FIGURE OF MERIT}

The quality of neutron-gamma discrimination for a given scintillator is characterized by figure of merit (FOM), see

Fig.

9.

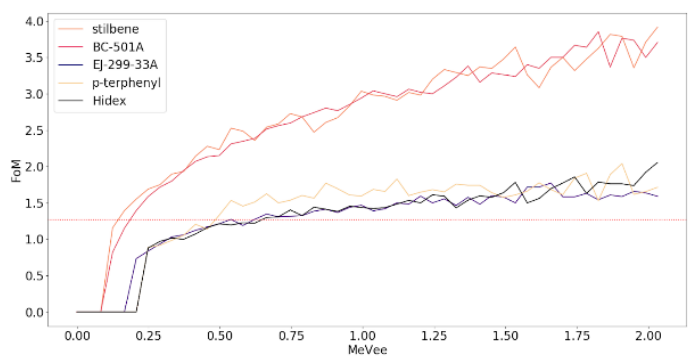

Fig. 9 FoMs for examined scintillators

Selected results of neutron-gamma separation quality are shown in Tab. 1. The critical value of FoM 1.27 is an indicator of good neutron-gamma separation [3]. Yellow fields indicate gamma energies where FoM values exceed the critical value. According to the combination of FoM and energy parameters we can evaluate the quality of neutron-gamma separation. Stilbene detector achieves the best quality of separation because the FoM exceeds the critical value at the smallest energy.

\begin{tabular}{l|lllllll} 
MeVee & 0,12 & 0,17 & 0,2 & 0,29 & 0,5 & 0,62 & 0,66 \\
& & & & & & & \\
\hline stilbene & 1,16 & 1,4 & 1,55 & 1,75 & 2,23 & 2,36 & 2,54 \\
BC-501A & 0,82 & 1,15 & 1,39 & 1,72 & 2,15 & 2,39 & 2,51 \\
$\begin{array}{l}\text { p- } \\
\text { terphenyl }\end{array}$ & 0 & 0 & 0 & 0,92 & 1,33 & 1,51 & 1,63 \\
EJ-299-33 & 0 & 0 & 0,73 & 0,94 & 1,21 & 1,27 & 1,35 \\
Hidex & 0 & 0 & 0 & 0,97 & 1,21 & 1,22 & 1,3
\end{tabular}

Among the examined scintillators the stilbene detector is considered to have the best energy resolution. Apparatus spectra from the two best scintillators from Tab. 1 were compared with stilbene detector in Fig 10. The X-axis contains channel number, $y$-axis contains ratio $E_{s} / E_{x}$, where $E_{s}$ is apparatus spectrum of stilbene and $\mathrm{E}_{\mathrm{x}}$ is a spectrum for $\mathrm{BC}$ 501A and p-terphenyl. 


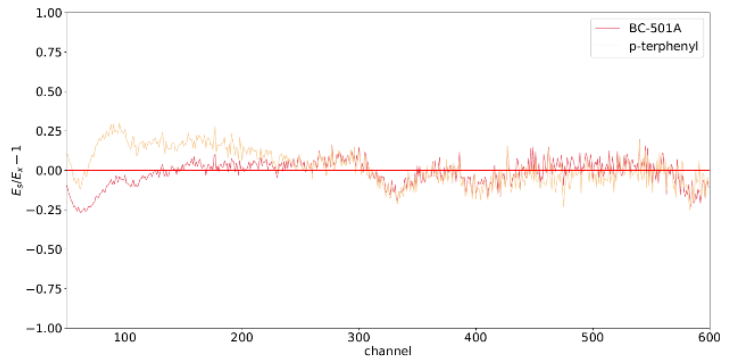

Fig. $10 \mathrm{E}_{\mathrm{s}} / \mathrm{E}_{\mathrm{x}}-1$ comparison of BC-501A and p-terphenyl with stilbene

\section{ACKNOWLEDGMENT}

The presented work has been supported by Ministry of Industry and Trade, within the project TRIO, No. FV20453. Presented results were obtained with the use of the infrastructure Reactors LVR-15 and LR-0, which is financially supported by the Ministry of Education, Youth and Sports - project LM2015074 and also financially supported by the Ministry of Education, Youth and Sports Czech Republic - project LQ1603 Research for SUSEN. This work has been realized within the SUSEN Project (established in the framework of the European Regional Development Fund (ERDF) in project CZ.1.05/2.1.00/03.0108 and of the European Structural Funds and Investment Funds (ESIF) in the project CZ.02.1.01/0.0/0.0/15_008/0000293), which is financially supported by the Ministry of Education, Youth and Sports - project LM2015093 Infrastructure SUSEN.

\section{REFERENCES}

[1] KOŠŤÁL, M., M. SCHULC, J. ŠOLTÉS, E. LOSA, L. VIERERBL, Zdeněk MATĚJ, F. CVACHOVEC a V. RYPAR. Measurements of neutron transport of well defined silicon filtered beam in lead. Applied Radiation and Isotopes, Elsevier, 2018, roč. 2018, č. 142, s. 160-166. ISSN 0969-8043.

[2] KOŠŤÁL, Michal, Jaroslav ŠOLTÉS, Ladislav VIERERBL, Zdeněk MATĚJ, František CVACHOVEC, Vojtěch RYPAR a Evžen LOSA. Measurement of Neutron Spectra in a silicon filtered neutron beam using stilbene detectors at the LVR-15 research reactor. Applied Radiation and Isotopes, 2017, roč. 128, s. 41-48.

[3] N. Zaitseva, B. L. Rupert, I. Pawełczak, A. Glenn, H. P. Martinez, L. Carman, M. Faust, N. Cherepy, S. Payne, Plastic scintillators with efficient neutron/gamma pulse shape discrimination, Nuclear Instruments and Methods in Physics Research Section A: Accelerators, Spectrometers, Detectors and Associated Equipment, Volume 668, 2012, Pages 88-93

[4] CVACHOVEC, Jiří a František CVACHOVEC. Maximum likelihood estimation of a neutron spectrum and associated uncertainties. Advances in Military Technology, Brno: Univerzita obrany, 2008, Vol. 3, No. 2. 